\title{
Approximate Design of Optimal Disturbance Rejection for Discrete-Time Systems with Multiple Delayed Inputs: Application to a Jacket-Type Offshore Structure
}

\author{
Shi-Yuan Han, ${ }^{1}$ Dong Wang, ${ }^{1}$ Yue-Hui Chen, ${ }^{1}$ Gong-You Tang, ${ }^{2}$ and Xi-Xin Yang ${ }^{3}$ \\ ${ }^{1}$ Shandong Provincial Key Laboratory of Network Based Intelligent Computing, University of Jinan, \\ 336 Nanxinzhuang Road, Jinan 250022, China \\ ${ }^{2}$ College of Information Science and Engineering, Ocean University of China, 238 Songling Road, Qingdao 266100, China \\ ${ }^{3}$ College of Software Technical, Qingdao University, Qingdao 266100, China
}

Correspondence should be addressed to Dong Wang; ise_wangd@ujn.edu.cn

Received 28 May 2014; Accepted 26 July 2014

Academic Editor: Hak-Keung Lam

Copyright (C) 2015 Shi-Yuan Han et al. This is an open access article distributed under the Creative Commons Attribution License, which permits unrestricted use, distribution, and reproduction in any medium, provided the original work is properly cited.

\begin{abstract}
The study is concerned with problem of optimal disturbance rejection for a class of discrete-time systems with multiple delayed inputs. In order to avoid the two-point boundary value (TPBV) problem with items of time-delay and time-advance caused by multiple delayed inputs, the discrete-time system with multiple delayed inputs is transformed into a delay-free system by introducing a variable transformation, and the original performance index is reformulated as a corresponding form without the explicit appearance of time-delay items. Then, the approximate optimal disturbance rejection controller (AODRC) is derived from Riccati equation and Stein equation based on the reduced system and reformulated performance index, which is combined with feedback item of system state, feedforward item of disturbances, and items of delayed inputs. Also, the existence and uniqueness of AODRC are proved, and the stability of the closed-loop system is analysed. Finally, numerical examples of disturbance rejection for jacket-type offshore structure and pure mathematical model are illustrated to validate the feasibility and effectiveness of the proposed approach.
\end{abstract}

\section{Introduction}

In practice, time-delays are inherent in various engineering systems. As an efficient control algorithm for the improvement of the efficiency, reliability, and scalability of the dynamic systems, time-delays must be taken into consideration in process of modeling and controller design from engineering and control scientists. It is well known that the actuators and transmission of the communication information are with the electrical and electromagnetic characteristics. Therefore, delayed inputs are more general pervasive on dynamic modelling of engineering systems. Especially, with the rise of networked control systems (NCSs) and communication systems $[1,2]$, multiple delayed inputs are mainly caused by the online data acquisition, filtering, calculating controller, and transmitting the control force signals from a computer to the actuator in engineering systems. Recently, many methods have been proposed to solve the problem for the time-delay discrete-time systems. For example, sliding mode control (SMC) is a widely used technique to handle matched disturbances by designing sliding surface and reaching motion controller for the time-delay process [3-5]. Smith predictor has the potential capability to achieve an improvement of set-point response and disturbance response [6,7]; observer based output feedback control is very powerful for systems with delayed input by using the predictor feedback while the state vectors are measurable or estimated online $[8,9]$; $H_{\infty}$ control has been intensively applied among the stability analysis, stabilization control, and robustness control for discrete-time systems with multiple delayed inputs $[10,11]$.

Disturbance rejection control has been viewed as a typical issue since the origins of control theory, and many researchers have been paying more attention to this field both academia and engineers. Many physical systems are subjected to persistent disturbances, such as offshore jacket platforms subjected to the waves $[12,13]$, helicopters subjected to the 
winds [14], and vehicle suspension systems subjected to the road roughness $[15,16]$. The external disturbances are often a source of poor performance of underlying control systems. The active disturbance rejection control has been viewed as an effective solution to solve the above problem. However, finding an explicit form of optimal control law for time-delay systems under disturbances remains difficult [17]. Therefore, some modification or improvement has been done in the conventional control schemes. For example, active disturbance rejection control has been shown to be an effective tool in dealing with real world problems of dynamic uncertainties, disturbances, and nonlinearities [18-20]; a robust $H_{\infty}$ guaranteed cost control law is designed for uncertain Markovian jump systems with distributed delays and input delays in [21]; by using an inertial actuator for suppression of multiple unknown and/or time-varying vibrations, an active vibration control system was proposed in [22]; a disturbance estimator design method was proposed to design the control scheme for open-loop and unstable processes with time delay [23]. An overview of antidisturbance control for engineering systems with multiple disturbances can be found in [24].

For discrete-time systems with multiple delayed input under persistent disturbances, the traditional optimal control problem with respect to quadratic performance index will induce (TPBV) problem with time-delay and timeadvance items. Although there have been many methods to solve the control problem for discrete-time systems with delayed input, it is difficult to find an optimal solution for this problem. On the one hand, augmented method could convert a delay system into free-delay ones. However, with the multiple delays or large delays, the dimension and stability of the delay-free system cannot be ensured; even the computing work would increase exponentially. On the other hand, virtually most of the studies on optimal control for discrete-time system with multiple input delays consider only approximate optimal control by using an iterative solution. For example, iterative learning algorithm was proposed to ensure the control performance for nonlinear discrete-time systems with multiple input delays in [25]; [26] proposed an optimal controller by using only measured input/output data from a class of linear discrete-time systems with multiple delays by using adaptive dynamic programming; a successive approximation approach (SAA) was proposed in [27] to design a suboptimal control for discrete linear systems with time delay, in which an iterative procedure is designed to solve the TPBV problem with time-delay items and nonlinear items; after that, based on SAA and augmented method, [28] designed an approximate tracking controller for discretetime systems with multiple state and input delays.

The motivation of this work is to design an AODRC for discrete-time systems with multiply delayed inputs under persistent disturbances with respect to a typical quadric performance index. In order to deal with multiple delayed inputs, a variable transformation is proposed so that the original system is transformed into delay-free ones, and the quadratic performance index of the original system is simplified without the explicit appearance of time-delay items. Then, the AODRC is designed based on the maximum principle by solving Riccati and Stein equations, which is composed of a feedback item of system state, a feedforward item of disturbance state, and some control memory terms. In term of design of controller, compared to the previous work $[25,27,28]$, the proposed AODRC is analytical solution so that the computation cost and time are reduced. In terms of control performance, by analysing the simulation results for an offshore platform and pure mathematical example with different cases in delayed inputs, the proposed AODRC can not only reduce the influence from multiple delayed inputs for underlying systems but also trade off among rejection effect opposed to the multiple delayed inputs, persistent disturbances, and energy consumption.

The rest of the paper is organized as follows. The disturbance rejection problem for discrete-time systems with multiple delayed inputs is formulated in Section 2. In Section 3, the original problem is reformulated as an equivalent one for delay-free transformed system with respect to a transformed performance index without the explicit appearance of time delay. Then, the proposed AODRC is presented in Section 4, in which its existence and uniqueness are proved, and the stability of system with multiple delayed inputs is analysed. In Section 5, the effectiveness and implement of the proposed AODRC are evaluated by applying it to numerical examples of pure mathematical example and a jacket-type offshore platform for different cases with delayed inputs. Finally, our findings are concluded in Section 6.

\section{Problem Statement}

Consider the discrete-time systems with multiple delayed inputs under persistent disturbances described by

$$
\begin{gathered}
x(k+1)=A x(k)+\sum_{i=1}^{N} B_{i} u\left(k-h_{i}\right)+D v(k), \\
y(k)=C x(k), \quad k=0,1,2, \ldots, \\
x(0)=x_{0}, \\
u(k)=0, \quad k<0,
\end{gathered}
$$

where $x \in R^{n}$ denotes the state vector; $u \in R^{m}$ is the control input; $v \in R^{p}$ denotes the persistent disturbances signal; $h_{i}>0(i=1,2, \ldots, N)$ are positive multiple delayed inputs, respectively; $y \in R^{r}$ is the output vector; $N$ is the account of multiple delayed inputs. $A, B_{i}(i=1,2, \ldots, N), C$, and $D$ are constant matrices of appropriate dimensions.

Assumption 1. The pair $\left(A, B_{1}\right)$ is completely controllable and the pair $(A, C)$ is completely observable, where $C$ is defined by $Q=C^{T} C$.

The dynamic characteristics of the persistent disturbances vector $v(k)$ are described by the following exosystem:

$$
\begin{gathered}
w(k+1)=G w(k) \\
v(k)=E w(k),
\end{gathered}
$$

where $w \in R^{q},(p \leq q \leq r)$ is the disturbance state, whose initial condition is unknown. $G$ and $E$ are constant matrices 
of appropriate dimensions with full rank. It is assumed that the pair $(E, G)$ is completely observable. Also, the eigenvalues of the $G$ satisfy

$$
\left|\lambda_{i}(G)\right| \leq 1, \quad i=1,2, \ldots, q
$$

Then, exosystem (2) of the persistent disturbances is stable but may not be asymptotically stable. It should be noted that the expression of exosystem (2) could describe the various forms of disturbances with known or unknown dynamic characteristics, such as the sinusoidal disturbances, periodic disturbances, step disturbances, and random signals.

The performance index could be depending on the dynamic characteristics of persistent disturbances. When the persistent disturbances are asymptotically stable, the traditional quadratic performance index could be chosen as

$$
J=\frac{1}{2} \sum_{k=0}^{\infty}\left[y^{T}(k) Q y(k)+u^{T}(k) R u(k)\right] .
$$

On the other hand, the persistent disturbances are stable but may not be asymptotically stable; the effect from persistent disturbance vector $v(k)$, the steady state of the state vector $x(k)$, and the control input $u(k)$ will not converge to zero synchronously. Therefore, the following infinite-time average performance index is chosen:

$$
J=\lim _{N \rightarrow \infty} \frac{1}{N} \sum_{k=0}^{N}\left[y^{T}(k) Q y(k)+u^{T}(k) R u(k)\right]
$$

where $Q \in R^{n \times n}$ is positive semidefinite and $R \in R^{m \times m}$ is positive definite.

The aim of the optimal disturbance rejection is to find an optimal control law $u^{*}(k)$ for the discrete-time systems described by (1) and (2) with respect to the performance index (4) or (5) that make the performance index (4) or (5) obtain the minimum value.

\section{Reformulation of Disturbance Rejection Problem}

For discrete-time delay systems with quadratic performance index, the optimal control problem is difficult to be solved analytically caused by the TPBV problem with both timedelay and time-advance terms. In this section, a vector transformation is introduced to deal with the multiple delayed inputs, and the performance index is reformulated as the equivalent ones without the explicit appearance of time delay.

3.1. Delay-Free Transformation of Control System. By proposing the following variable transformation

$$
\tilde{x}(k)=x(k)+\sum_{i=1}^{N} \sum_{j=k-h_{i}}^{k-1} A^{k-h_{i}-j-1} B_{i} u(j)
$$

system (1) with multiple delayed input can be transformed into the following form:

$$
\begin{gathered}
\tilde{x}(k+1)=A \tilde{x}(k)+\widetilde{B} u(k)+D v(k), \\
y(k)=C\left(\tilde{x}(k)-\sum_{i=1}^{N} \sum_{j=k-h_{i}}^{k-1} A^{k-h_{i}-j-1} u(j)\right),
\end{gathered}
$$

where $\tilde{x}(k) \in R^{n}$ is the state vector of the delay-free system (7) and $\widetilde{B}=\sum_{i=1}^{N} A^{-h_{i}} B_{i}$. Note that the transformed system (7) is in the form of a delay-free system, and the pair $(A, \widetilde{B})$ is completely controllable if and only if $\left(A, B_{1}\right)$ is completely controllable [29].

3.2. Simplification of Performance Index. Through the vector transformation (6) introduced, the original system with multiple delayed inputs (1) could be transformed into system (7) without the explicit appearance of time delay. In order to evaluate the effectiveness of the control law more exactly, it is necessary to transform the performance index (4) or (5) into the equivalent form for the transformed system (7).

By using (6) and (7), the quadratic performance index (4) could be transformed into the following form:

$$
\begin{array}{r}
J=\sum_{k=0}^{\infty}\left\{\tilde{x}^{T}(k) C^{T} Q C \tilde{x}(k)+u^{T}(k) R u(k)\right. \\
\left.-2 U_{1}^{T} C^{T} Q C \tilde{x}(k)+U_{1}^{T} C^{T} Q C U_{1}\right\},
\end{array}
$$

where $U_{1}=\sum_{i=1}^{N} \sum_{j=k-h_{i}}^{k-1} A^{k-h_{i}-j-1} u(j)$.

Based on (2), (6), (7), and (9), one gets

$$
\begin{gathered}
\sum_{k=0}^{\infty} \tilde{x}^{T}(k) C^{T} Q C U_{1} \\
=\sum_{k=0}^{\infty}\left\{\tilde{x}^{T}(k) C_{1} u(k)+w^{T}(k) C_{2} u(k)\right. \\
+\sum_{t=1}^{N} \sum_{i=1}^{h_{t}} \sum_{j=1}^{h_{t}}\left(\left(C A^{-j} B_{i} u(k+i-1)\right)^{T}\right. \\
\left.\left.\times Q_{k=0}^{\infty} C^{T} Q C A^{i-j-1} B_{i}\right) u(k)\right\} \\
=\sum_{k=0}^{\infty}\left\{\begin{array}{c}
u^{T}(k) \sum_{i=1}^{N} \sum_{j=1}^{h_{i}}\left(\left(C A^{-j} B_{i}\right)^{T} Q C A^{-j} B_{i}\right) u(k) \\
+2 \sum_{t=1}^{N} \sum_{i=1}^{h_{t}} \sum_{j=1}^{h_{t}}\left(\left(C A^{-j} B_{i} u(k+i-1)\right)^{T}\right.
\end{array}\right.
\end{gathered}
$$


where

$$
\begin{aligned}
& C_{1}=\sum_{i=1}^{N}\left(\left(\sum_{j=1}^{h_{i}}\left(C A^{j}\right)^{T} \mathrm{Q} A C A^{j-1-h_{i}} B_{i}\right)\right), \\
& C_{2}=\sum_{i=1}^{N} \sum_{j=1}^{h_{i}} \sum_{t=0}^{h_{i}-j}\left(\left(C A^{t} D E G^{j-1}\right)^{T} Q C A^{t+j-h_{i}-1} B_{i}\right) .
\end{aligned}
$$

Then, the quadratic performance index (4) could be reformed as the following form:

$$
\begin{array}{r}
J=\frac{1}{2} \sum_{k=0}^{\infty}\left\{\widetilde{x}^{T}(k) C^{T} Q C \widetilde{x}(k)+u^{T}(k) \widetilde{R} u(k)\right. \\
\left.-2 \widetilde{x}^{T}(k) C_{1} u(k)-2 w^{T}(k) C_{2} u(k)\right\},
\end{array}
$$

where $\widetilde{R}=R+\sum_{i=1}^{N} \sum_{j=1}^{h_{i}}\left(\left(C A^{-j} B_{i}\right)^{T} Q C A^{-j} B_{i}\right)$.

Actually, the above transformations are suitable for the infinite-time average quadratic performance index (5). The reformed performance index could be described as

$$
\begin{aligned}
J=\lim _{N \rightarrow \infty} \frac{1}{N} \sum_{k=0}^{N}\left\{\tilde{x}^{T}(k) C^{T} Q C \tilde{x}(k)+u^{T}(k) \widetilde{R} u(k)\right. \\
\left.-2 \tilde{x}^{T}(k) C_{1} u(k)-2 w^{T}(k) C_{2} u(k)\right\} .
\end{aligned}
$$

Then, the original optimal disturbance rejection is reformulated as an equivalent optimal regulation problem that designs an approximate control law $u^{*}(k)$ to make the quadratic performance index (11) or (12) obtain the minimum value subject to the discrete-time system (2) and (7) such that the negative effects from persistent disturbances and multiple delayed inputs are eliminated.

\section{Approximate Design of Optimal Disturbance Rejection Controller}

In this section, the proposed AODRC is presented in detail, and its existence and uniqueness are proved. In order to state the proposed control law clearly, the following matrices are defined:

$$
\begin{gathered}
A_{1}=A+\widetilde{B} \widetilde{R}^{-1} C_{1}^{T}, \quad A_{2}=D E+\widetilde{B} \widetilde{R}^{-1} C_{2}^{T}, \\
Q_{1}=C^{T} Q C-C_{1} \widetilde{R}^{-1} C_{1}^{T}, \quad Q_{2}=C_{1} \widetilde{R}^{-1} C_{2}^{T} .
\end{gathered}
$$

Theorem 2. Consider the optimal disturbance rejection control problem for the discrete-time systems given by (1) and (2) with respect to the quadratic performance indexes (4) or
(5). The approximate optimal disturbance rejection controller uniquely exists and can be formulated as

$$
\begin{aligned}
u^{*}(k)=\widetilde{R}^{-1}\{ & {\left[C_{1}^{T}-\widetilde{B}^{T} A_{1}^{-T}\left(P_{1}-Q_{1}\right)\right] } \\
\times & {\left[x(k)+\sum_{i=1}^{N} \sum_{j=k-h_{i}}^{k-1} A^{k-h_{i}-j-1} B u(j)\right] } \\
+ & \left.\left(C_{2}^{T}-\widetilde{B}^{T} A_{1}^{-T}\left(P_{2}+Q_{2}\right)\right) w(k)\right\},
\end{aligned}
$$

where $P_{1}$ is the unique positive definite solution of the Riccati matrix equation

$$
P_{1}=Q_{1}+A_{1}^{T} P_{1}\left(I+\widetilde{B} \widetilde{R}^{-1} \widetilde{B}^{T} P_{1}\right)^{-1} A_{1}
$$

and $P_{2}$ is the unique solution of the Stein matrix equation

$$
\begin{aligned}
P_{2}+Q_{2}= & A_{1}^{T} P_{1}\left(I+\widetilde{B} \widetilde{R}^{-1} \widetilde{B}^{T} P_{1}\right)^{-1} A_{2} \\
& +A_{1}^{T}\left[I-A_{1}^{T} P_{1}\left(I+\widetilde{B} \widetilde{R}^{-1} \widetilde{B}^{T} P_{1}\right)^{-1} \widetilde{B} \widetilde{R}^{-1} \widetilde{B}^{T}\right] P_{2} G .
\end{aligned}
$$

Proof. Define the Hamiltonian function for the optimal disturbance rejection problem as

$$
\begin{aligned}
H(\bullet)= & \frac{1}{2}\left\{\tilde{x}^{T}(k) C^{T} Q C \tilde{x}(k)+u^{T}(k) \widetilde{R} u(k)\right. \\
& \left.-2 \widetilde{x}^{T}(k) C_{1} u(k)-2 w^{T}(k) C_{2} u(k)\right\} \\
+ & \lambda^{T}(k+1)[A \widetilde{x}(k)+\widetilde{B} u(k)+D v(k)] .
\end{aligned}
$$

Applying the maximum principle condition $\partial H / \partial u=0$ to this specific Hamiltonian function (17) yields

$$
u(k)=\widetilde{R}^{-1}\left[\widetilde{B}^{T} \lambda(k+1)-C_{1}^{T} \tilde{x}(k)-C_{2}^{T} w(k)\right],
$$

where $\lambda(k)$ is the solution of the following TPBV problem:

$$
\begin{gathered}
\tilde{x}(k+1)=A_{1} \widetilde{x}(k)+A_{2} w(k)-\widetilde{B} \widetilde{R}^{-1} \widetilde{B}^{T} \lambda(k+1), \\
Q_{1} \widetilde{x}(k)-Q_{2} w(k)+A_{1}^{T} \lambda(k+1)=\lambda(k), \\
\widetilde{x}(0)=x(0), \\
\lambda(\infty)=0 .
\end{gathered}
$$

To solve TPBV problem (19), let

$$
\lambda(k)=P_{1} \tilde{x}(k)+P_{2} w(k) .
$$

Rearranging (19), one gets

$$
\begin{aligned}
\lambda(k+1)= & A_{1}^{-T}\left[\left(P_{1}-Q_{1}\right) \widetilde{x}(k)+\left(P_{2}+Q_{2}\right) w(k)\right], \\
x(k+1)= & \left(I+\widetilde{B} \widetilde{R}^{-1} \widetilde{B}^{T} P_{1}\right)^{-1} \\
& \times\left\{A_{1} x(k)+\left(A_{2}-\widetilde{B} \widetilde{R}^{-1} \widetilde{B}^{T} P_{2} G\right) w(k)\right\} .
\end{aligned}
$$


Substituting the first formula of (21) into (18), the approximate optimal control law (14) is obtained. Note that

$$
\begin{aligned}
\lambda(k)= & Q_{1} \widetilde{x}(k)-Q_{2} w(k)+A_{1}^{T} \lambda(k+1) \\
= & {\left[Q_{1}+A_{1}^{T} P_{1}\left(I+\widetilde{B} \widetilde{R}^{-1} \widetilde{B}^{T} P_{1}\right)^{-1} A_{1}\right] \widetilde{x}(k) } \\
+ & \left\{A_{1}^{T}\left[I-A_{1}^{T} P_{1}\left(I+\widetilde{B} \widetilde{R}^{-1} \widetilde{B}^{T} P_{1}\right)^{-1} \widetilde{B} \widetilde{R}^{-1} \widetilde{B}^{T}\right] P_{2} G\right. \\
& \left.+A_{1}^{T} P_{1}\left(I+\widetilde{B} \widetilde{R}^{-1} \widetilde{B}^{T} P_{1}\right)^{-1} A_{2}-Q_{2}\right\} w(k) .
\end{aligned}
$$

By comparing the parameters of (20) and (22), the Riccati equation (15) and Stein equation (16) can be obtained.

In the following, the existence and uniqueness of the proposed controller will be proved. Because $P_{1}$ and $P_{2}$ are unknown in the parameters of proposed control law (14), the existence and uniqueness of (14) are equivalent to that of $P_{1}$ and $P_{2}$. For Riccati matrix equation (15), because of the completely controllable and observable pairs $\left(A, B_{1}\right)$ and $(A, C)$, the matrix $P_{1}$ is existent and the unique solution of Riccati matrix equation (15). It should be noted that the first formula of (19) is equivalent to (7); based on the completely controllable pair $(A, \widetilde{B})$, one gets

$$
\left|\lambda_{i}\left\{\left[I+\widetilde{B} \widetilde{R}^{-1} \widetilde{B}^{T} P_{1}\right]^{T} A_{1}\right\}\right|<1, \quad i=1,2, \ldots, n .
$$

Note (3); one gets

$$
\begin{array}{r}
\left|\lambda_{i}\left\{\left[I+\widetilde{B} \widetilde{R}^{-1} \widetilde{B}^{T} P_{1}\right]^{T} A_{1}\right\}\right|\left|\lambda_{j}(G)\right|<1, \\
i=1,2, \ldots, n, \quad j=1,2, \ldots, q .
\end{array}
$$

Then, $P_{2}$ is existent and the unique solution of Stein matrix equation (16). Therefore, the proposed approximate controller is existent and unique.

According to [29], (6), and (14), one gets

$$
\begin{gathered}
\|x(k)\| \leq\|\widetilde{x}(k)\|+\sum_{i=1}^{N}\left[h_{i} \max _{1 \leq s \leq h_{i}}\left\|A^{k-j-1}\right\|\right. \\
\times\left\|B \widetilde{R}^{-1}\left[\widetilde{B}^{T} A_{1}^{-T}\left(P_{1}-Q_{1}\right)-C_{1}^{T}\right]\right\| \\
\left.\times\|\widetilde{x}(k-s)\| \max _{1 \leq s \leq h_{i}}\right] \\
+\sum_{i=1}^{N}\left[h_{i} \max _{1 \leq s \leq h_{i}}\left\|A^{k-j-1}\right\|\|w(k-s)\|\right. \\
\left.\quad \times\left\|B \widetilde{R}^{-1}\left[\widetilde{B}^{T} A_{1}^{-T}\left(P_{2}+Q_{2}\right)-C_{2}^{T}\right]\right\|\right] .
\end{gathered}
$$

Because $\|\widetilde{x}(k)\|$ and $\|w(k)\|$ are bounded, $\|x(k)\|$ is bounded. Then, the proposed control law (14) is a stabilizing control law for discrete-time system (1) with multiple delayed inputs. The proof is completed.
TABLE 1: Parameters of installed AMD device.

\begin{tabular}{lccc}
\hline Name of parameter & Variable & Value & Unit \\
\hline Mass & $m_{2}$ & 11855 & $\mathrm{~kg}$ \\
Natural frequency & $\omega_{2}$ & 2.33 & $\mathrm{rad} / \mathrm{s}$ \\
Structural damping ratio & $\xi_{2}$ & 9.32 & Percent \\
\hline
\end{tabular}

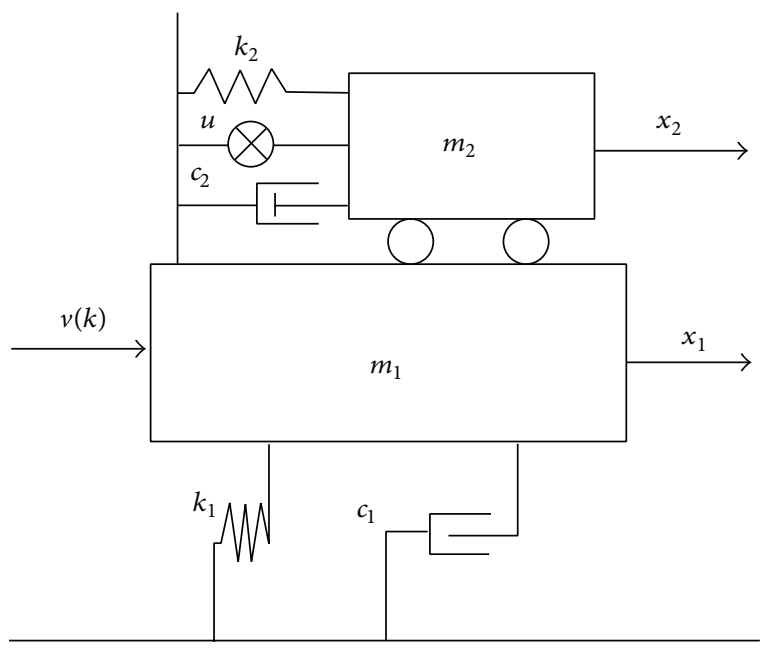

FIGURE 1: The sketch of offshore platform with AMD device.

\section{Simulation}

In order to demonstrate the effectiveness and feasibility of the proposed AODRC, numerous simulations are undertaken in this section. In case 1 , the vibration control problem for offshore steel jacket platforms with single delayed input is simulated by using the proposed AODRC. In case 2, the disturbance rejection problem for pure mathematical example with two delayed inputs is considered.

5.1. Vibration Control for Offshore Jacket Platforms with Single Delayed Input. The jacket type platforms play an import role in the oil exploration and drilling operations. However, the jacket type platforms subject to external disturbances persistently, such as wave force, wind, and earthquake. In order to ensure the safety and production efficiency, the vibration caused by external disturbances could be rejected effectively, especially for wave force. In this subsection, the vibration control problem for offshore jacket platforms with single input delay is solved by the proposed control law.

Consider the jacket platform located in Bohai Bay, the sketch of offshore platform with an AMD device is shown in Figure 1. The structural parameters of offshore platform and installed AMD device on the deck of the jacket type platform are listed in Tables 1 and 2 [30], respectively.

Based on the JONSWAP spectrum, the wave can be described as

$$
S_{\eta}(\omega)=\left[\frac{5 H_{s}^{2}}{16 \omega_{0}}\right]\left(\frac{\omega_{0}}{\omega}\right)^{5} \exp \left[-1.25\left(\frac{\omega_{0}}{\omega}\right)^{-4}\right] \gamma^{\beta},
$$


TABLE 2: Parameters of offshore platform.

\begin{tabular}{lccc}
\hline Name of parameter & Variable & Value & Unit \\
\hline Equivalent characteristic diameter of legs & $D$ & 1.7 & $\mathrm{~m}$ \\
First modal mass & $m_{1}$ & 2371100 & $\mathrm{~kg}$ \\
Natural frequency & $\omega_{1}$ & 2.20 & $\mathrm{rad} / \mathrm{s}$ \\
Structural damping ratio & $\xi_{1}$ & 4 & Percent \\
Shape function of first mode & $\varphi(s)$ & $1-\cos \left(\frac{\pi s}{2 L}\right)$ & $0 \leq s \leq L$ \\
\hline
\end{tabular}

where $H_{s}=4 \mathrm{~m}$ is the significant wave height, $\omega$ is the wave frequency, $\gamma=3.3$ is the peakedness parameter, $\omega_{0}=0.87 \mathrm{rad} / \mathrm{s}$ is the peak frequency, $\beta=$ $\exp \left[-\left(\omega-\omega_{0}\right)^{2} /\left(2 \sigma^{2} \omega_{0}^{2}\right)\right]$, in which $\sigma$ is the shape parameter, $\sigma=0.07\left(\omega \leq \omega_{0}\right)$, and $\sigma=0.09\left(\omega>\omega_{0}\right)$. Then, based on [30], the wave force can be formulated as an exosystem described by (2) with the water depth $d=13.2 \mathrm{~m}$, the drag coefficient $C_{d}=1.2$, and the inertial coefficient $C_{m}=2.0$. The external disturbances from irregular wave force are displayed in Figure 2.

By setting the sampling period $T=0.1 \mathrm{~s}, Q=$ $\operatorname{diag}\left(\begin{array}{llll}100 & 0 & 100 & 0\end{array}\right)$, and $R=1$, the discrete-time model of the offshore structure could be established with the same expression of (1) based on the previous investigations on the subject, which is described as

$$
\begin{aligned}
& A=\left[\begin{array}{cccc}
0.9790 & 0.0002 & 0.0989 & 0.0000 \\
0.0205 & 0.9789 & 0.0047 & 0.0952 \\
-0.4182 & 0.0041 & 0.9701 & 0.0010 \\
0.3954 & -0.4152 & 0.0996 & 0.8993
\end{array}\right] \\
& B=\left[\begin{array}{c}
-0.0006 \\
0.0621 \\
-0.0120 \\
1.2205
\end{array}\right] \\
& D=\left[\begin{array}{l}
0.0006 \\
0.0000 \\
0.0126 \\
0.0006
\end{array}\right] \text {, } \\
& C=\left[\begin{array}{llll}
1 & 0 & 0 & 0 \\
0 & 0 & 1 & 0
\end{array}\right] \text {. }
\end{aligned}
$$

According to the previous investigations on the subject, the range of delayed input is in the interval $(0,1) \mathrm{s}$. Therefore, to demonstrate the effectiveness of the proposed AODRC, the comparison results between the open-loop system, the feedback control, and the AODRC with single delayed input $h=6$ are compared, in which feedback control law is just considered the feedback item in AODRC. Then, the curves of displacement of offshore structure, velocity of offshore structure, and control law are presented in Figures 3, 4, and 5 , respectively.

In order to show the comparison results among the simulated case clearly, the values of the quadratic performance index with different delayed input are compared in Table 3. Note that the percentage number given in the parentheses indicates the reduced amount of the closed-loop response

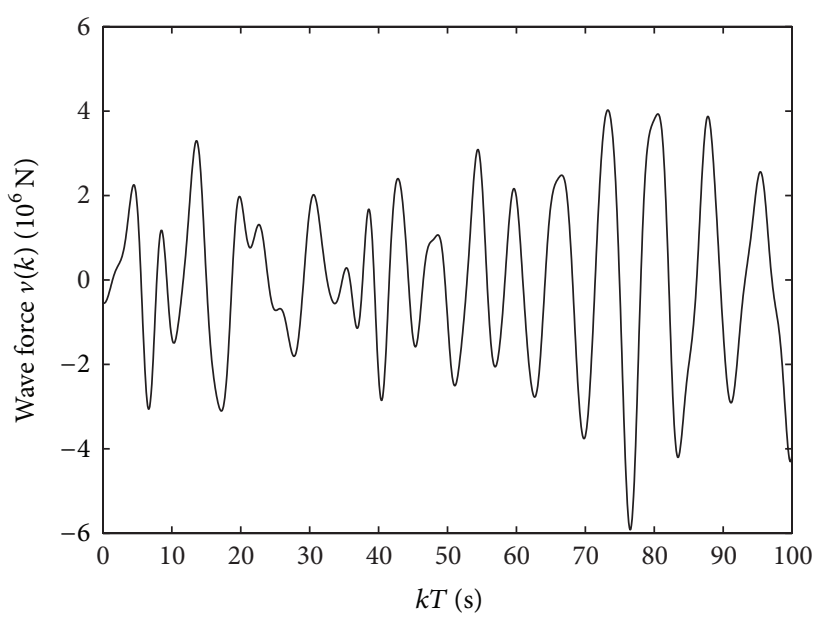

FIgURE 2: The external disturbances of wave force.

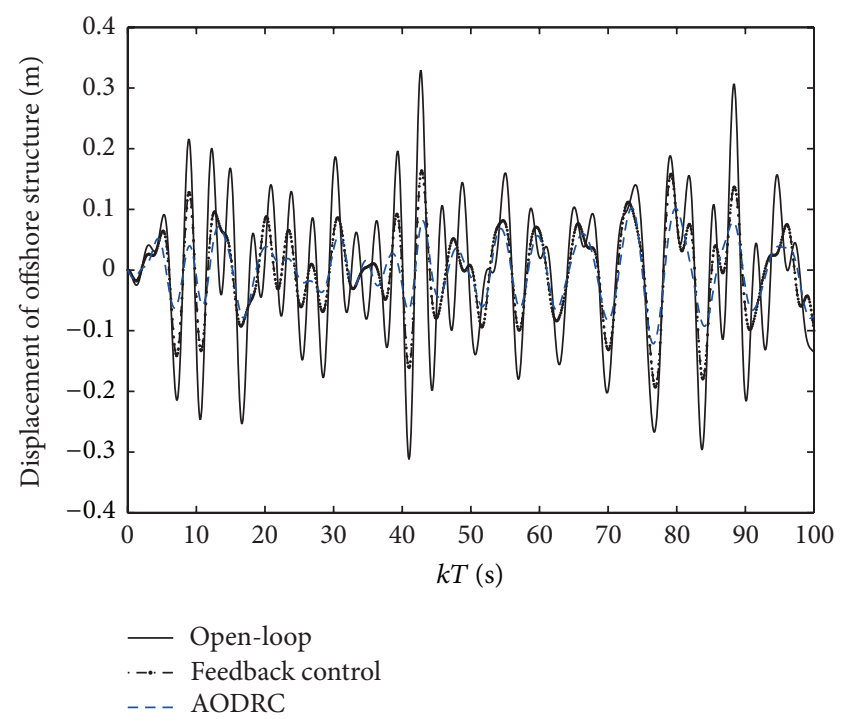

FIgURE 3: Curves of the displacement of offshore structure.

relative to the open-loop case. For instance, the value of performance index is reduced by $18.40 \%$ when delayed input $h=2$ less than that of open-loop one, using feedback control, while the value of performance index is reduced by $67.23 \%$ less than that of open-loop one by using proposed AODRC. 
TABLE 3: Comparison of values of performance index with different delayed input.

\begin{tabular}{lcccc}
\hline & $h=2$ & $h=4$ & $h=6$ & $h=8$ \\
\hline Open-loop & 5.0953 & 5.0953 & 5.0953 & 5.0953 \\
Feedback Control & $4.1578(18.40 \%)$ & $3.5232(30.85 \%)$ & $3.0731(39.69 \%)$ & $3.1232(38.70 \%)$ \\
AODRC & $1.6695(67.23 \%)$ & $1.6875(66.88 \%)$ & $1.7076(66.49 \%)$ & $1.7366(65.92 \%)$ \\
\hline
\end{tabular}

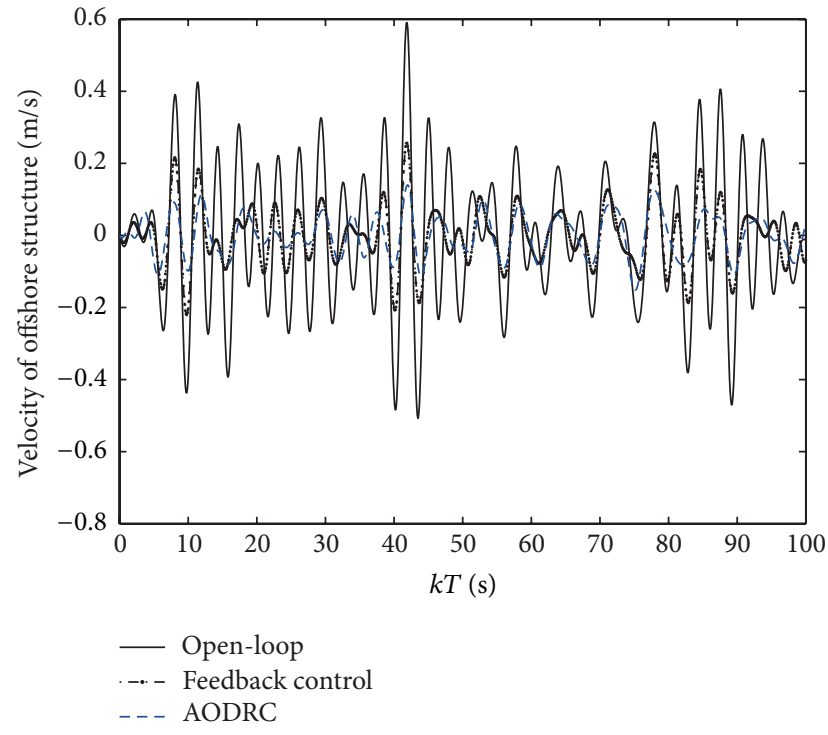

FIGURE 4: Curves of the velocity of offshore structure.

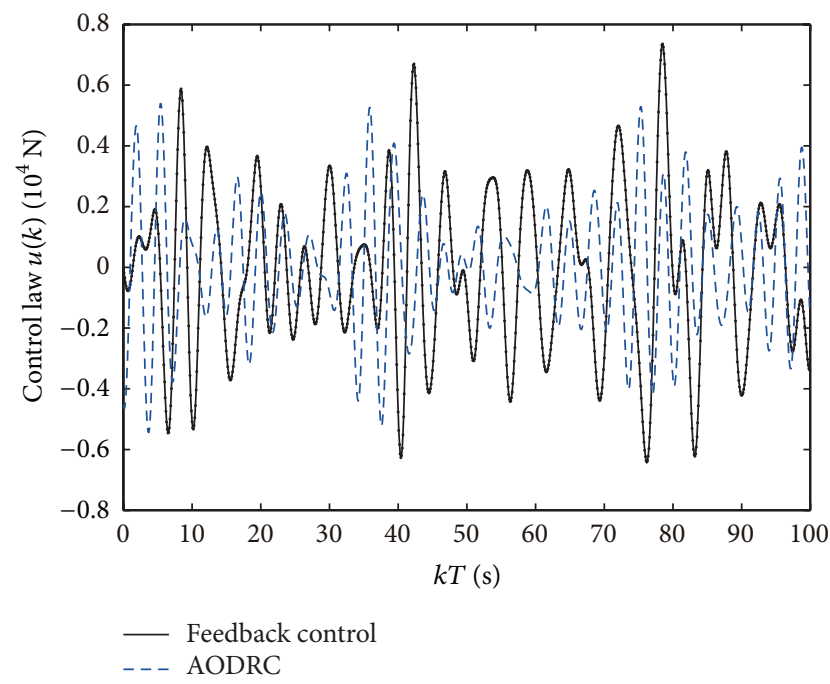

FIgURE 5: Curves of the control law.

In order to present the control results more intuitive, the maximum displacement, the maximum acceleration of offshore structure, and the maximum control law with different input delay are shown in Table 4. From Table 4, it can be seen clearly that the maximum displacement, the maximum acceleration of offshore structure, and the values of maximum control law with different input delay are close. The results show that the proposed control law could effectively eliminate the influences from different input delay and wave forces with high value.

It can be seen from Figures 3-5 and Tables 3-4 that the disturbances of wave force for offshore structure is reduced significantly by using the proposed AODRC. With the increase of delayed input, the proposed AODRC is still able to effectively control the displacement and velocity of offshore structure. What's more, the quadratic performance index is reduced significantly by using the proposed AODRC compared by the feedback control and open-loop ones. Therefore, we can conclude that the proposed AODRC is efficient to reject the disturbances from wave force for offshore structure, as well as being with lower consumption.

5.2. Disturbance Rejection for Pure Mathematical Example with Two Delayed Inputs. In order to verify the elasticity of the proposed AODRC, the discrete-time system with delayed inputs $h_{1}$ and $h_{2}$ under sinusoidal disturbances is considered. The matrices of (1) are chosen as

$$
\begin{aligned}
A & =\left[\begin{array}{cc}
1 & 0.4 \\
-0.2 & 0.9
\end{array}\right], \\
D & =\left[\begin{array}{l}
0 \\
1
\end{array}\right], \\
B_{1} & =\left[\begin{array}{l}
0.2 \\
0.5
\end{array}\right], \\
B_{2} & =\left[\begin{array}{c}
0.1 \\
0.15
\end{array}\right], \\
C & =\left[\begin{array}{ll}
1 & 0
\end{array}\right], \\
x(0) & =\left[\begin{array}{ll}
2 & 0
\end{array}\right]^{T} .
\end{aligned}
$$

The external disturbance $v(t)$ is chosen as sinusoidal disturbance; the matrices of (2) are given by

$$
\begin{aligned}
G & =\left[\begin{array}{cc}
0.4 \sqrt{5} & 0.4 \\
-0.5 & 0.4 \sqrt{5}
\end{array}\right], \\
E & =\left[\begin{array}{ll}
0.2 & 0
\end{array}\right], \\
v(0) & =\left[\begin{array}{ll}
1 & 0
\end{array}\right]^{T} .
\end{aligned}
$$

The curves of sinusoidal disturbances with (29) are shown in Figure 6. Also, the quadratic performance index is chosen as (5) with $Q=1, R=2$.

Then, the responses of system states $x_{1}$ and $x_{2}$ for different cases (open-loop, feedback control only, and proposed AODRC) with delayed inputs $h_{1}=1$ and $h_{2}=4$ are 
TABLE 4: The maximum displacement, the maximum acceleration of offshore structure, and the maximum control law.

\begin{tabular}{lcccc}
\hline & $h=2$ & $h=4$ & $h=6$ & $h=8$ \\
\hline $\begin{array}{l}\text { Maximum } \\
\text { displacement }(\mathrm{m})\end{array}$ & 0.126 & 0.130 & 0.146 & 0.150 \\
$\begin{array}{l}\text { Maximum } \\
\text { acceleration }\left(\mathrm{m}^{2} / \mathrm{s}\right)\end{array}$ & 0.171 & 0.175 & 0.178 & 0.181 \\
$\begin{array}{l}\text { Maximum control } \\
\text { input }\left(10^{4} \mathrm{~N}\right)\end{array}$ & 0.520 & 0.523 & 0.582 & 0.588 \\
\hline
\end{tabular}

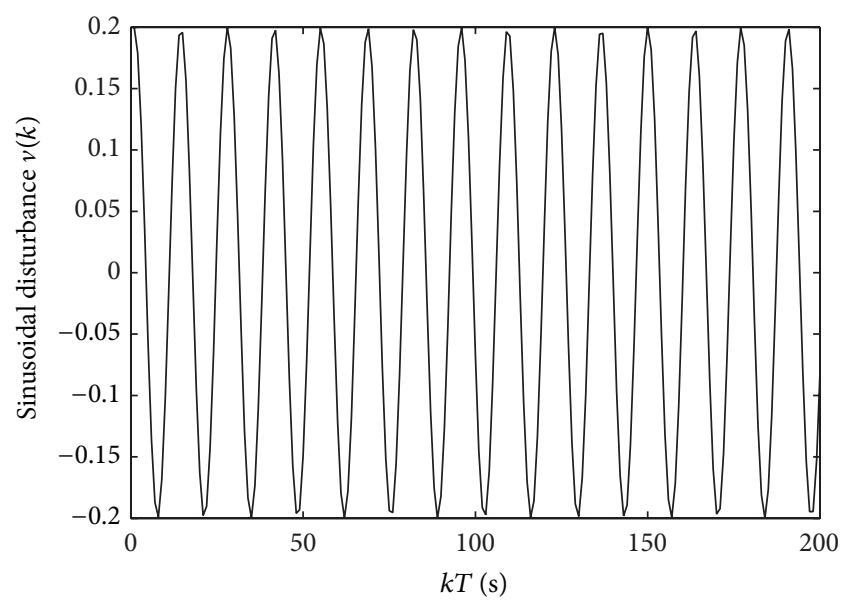

Figure 6: Curves of the sinusoidal disturbance $v(k)$.

simulated and shown in Figures 7 and 8. The curves of the proposed AODRC for different cases (the proposed AODRC and feedback control only) are shown in Figure 9. The values of quadratic performance index (5) are shown in Table 3 with two delayed inputs $\left(h_{1}=1, h_{2}=2 ; h_{1}=1, h_{2}=4 ; h_{1}=2\right.$, $h_{2}=4$ ). Figures 7 and 8 and Table 5 show that the proposed AODRC is able to effectively ensure the system states in lower values and reject the disturbances from external sinusoidal input.

From Figures 1-9 and Tables 1-5, the simulation results show that the proposed AODRC for the offshore platform and pure mathematical example are effective to attenuate the vibration amplitudes of the system state, and the quadratic performance index of the controlled system under AODRC is smaller than those under the open-loop and feedback control only. Moreover, the proposed AODRC is more scalable for different cases with multiple delayed inputs.

\section{Conclusion}

This paper has presented an interesting approach to solve the disturbance rejection problem for discrete-time systems with multiple delayed inputs under persistent external disturbances. A discrete-time system with multiple input delays is transformed into a non-delayed system in form, and the quadratic performance index has been transformed into a relevant format without the explicit appearance of time delay. Then, the AODRC is deployed to eliminate the negative effects from multiple delayed inputs and persistent external

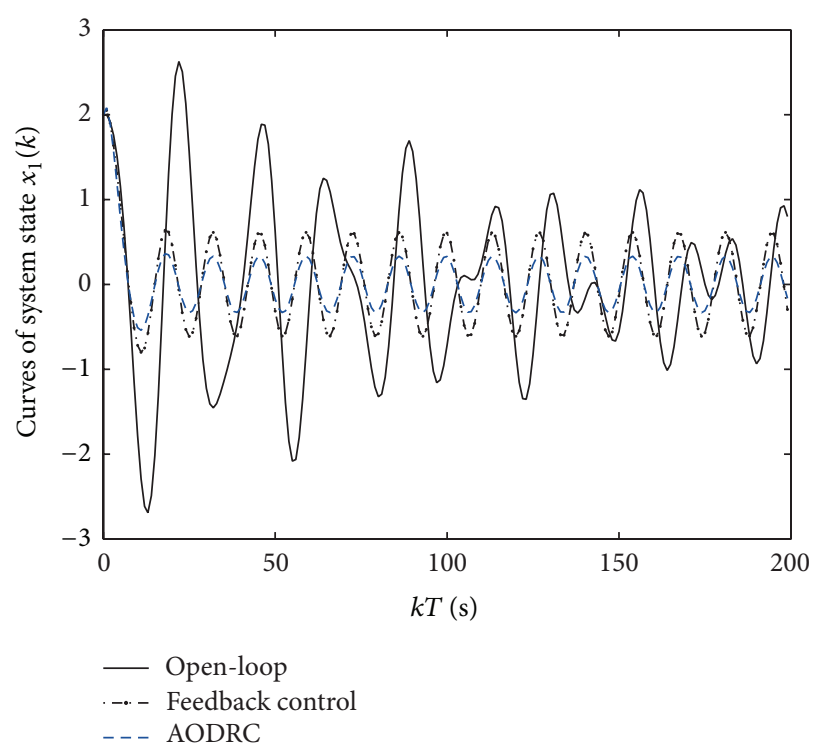

FIGURE 7: Curves of system state $x_{1}(k)$.

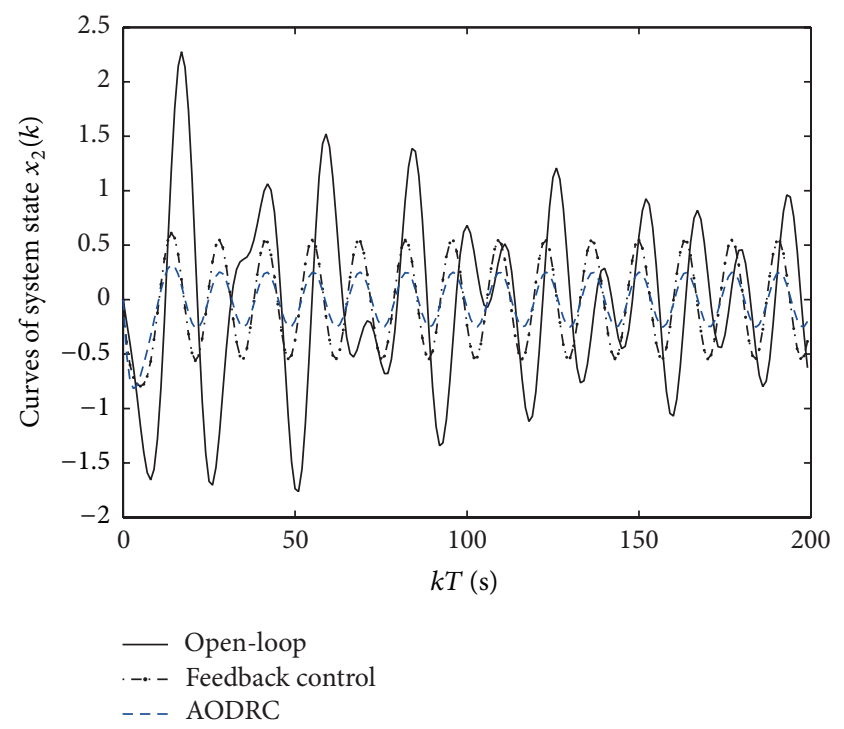

FIGURE 8: Curves of system state $x_{2}(k)$.

TABLE 5: Comparison of values of performance index with two delayed inputs.

\begin{tabular}{lccc}
\hline & $h_{1}=1, h_{2}=2$ & $h_{1}=1, h_{2}=4$ & $h_{1}=2, h_{2}=4$ \\
\hline $\begin{array}{l}\text { Open-loop } \\
\text { Feedback }\end{array}$ & 0.3422 & 0.3422 & 0.3422 \\
Control & $0.0836(75.57 \%)$ & $0.1055(69.17 \%)$ & $0.1035(69.75 \%)$ \\
AODRC & $0.0372(89.13 \%)$ & $0.0506(85.21 \%)$ & $0.0901(66.49 \%)$ \\
\hline
\end{tabular}

disturbances. On the other hand, the disturbance rejection problem for jacket-type offshore structure is illustrated to validate the feasibility and effectiveness of the proposed approach. 


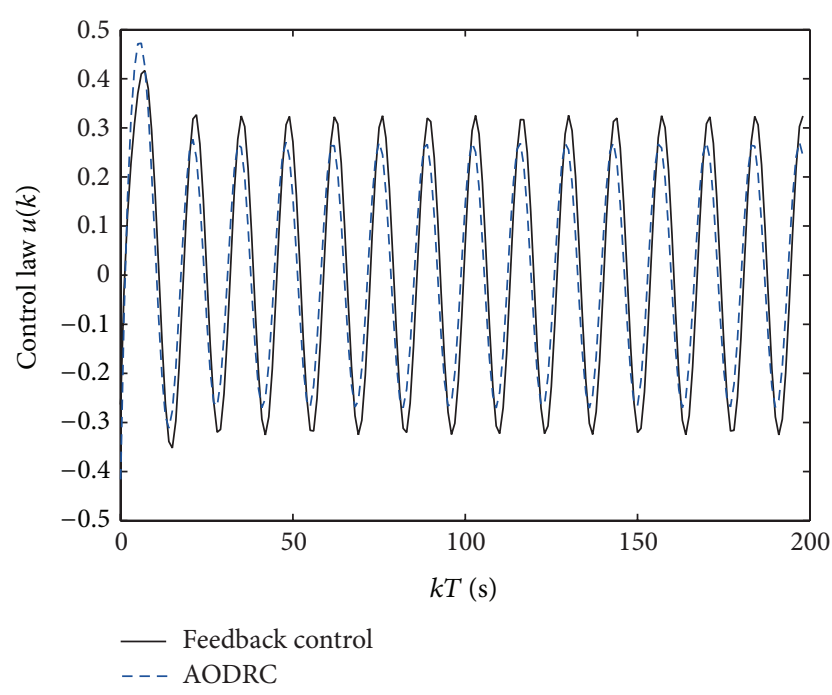

Figure 9: Curves of the control law.

\section{Conflict of Interests}

The authors declare that there is no conflict of interests regarding the publication of this paper.

\section{Acknowledgments}

This work was supported by the Program for Scientific Research Innovation Team in Colleges and Universities of Shandong Province, Science Foundation of China under Grants 61201428, 61203105, and 61302128, the Natural Science Foundation of Shandong Province under Grants ZR2011FZ003, ZR2012FQ016, and ZR2011FL022, and the Doctoral Foundation of Shandong Province under Grant BS2014DX015.

\section{References}

[1] E. Altman and T. Basar, "Optimal rate control for high speed telecommunication networks," in Proceedings of the 34th IEEE Conference on Decision and Control, vol. 2, pp. 1389-1394, December 1995.

[2] H. Zhang, L. Xie, and G. Duan, " $H_{\infty}$ control of discretetime systems with multiple input delays," IEEE Transactions on Automatic Control, vol. 52, no. 2, pp. 271-283, 2007.

[3] X. Yu, C. Wu, F. Liu, and L. Wu, "Sliding mode control of discrete-time switched systems with time-delay," Journal of the Franklin Institute, vol. 350, no. 1, pp. 19-33, 2013.

[4] S. Janardhanan and B. Bandyopadhyay, "Output feedback discrete-time sliding mode control for time delay systems," IEE Proceedings-Control Theory and Applications, vol. 153, no. 4, pp. 387-396, 2006.

[5] M. Cedomir, P.-D. Branislava, and V. Boban, "Discrete-time velocity servo system design using sliding mode control approach with disturbance compensation," IEEE Transactions on Industrial Informatics, vol. 9, no. 2, pp. 920-927, 2013.

[6] P. Albertos and P. García, "Predictor-observer-based control of systems with multiple input/output delays," Journal of Process Control, vol. 22, no. 7, pp. 1350-1357, 2012.
[7] E. Normey-Rico and E. F. Camacho, "Dead-time compensators: a survey," Control Engineering Practice, vol. 16, no. 4, pp. 407428, 2008.

[8] B. Zhou, Z.-Y. Li, and Z. Lin, "Observer based output feedback control of linear systems with input and output delays," Automatica, vol. 49, no. 7, pp. 2039-2052, 2013.

[9] B. Zhou, "Observer-based output feedback control of discretetime linear systems with input and output delays," International Journal of Control, 2014, in press.

[10] H. Zhang, G. Duan, and L. Xie, "Linear quadratic regulation for linear time-varying systems with multiple input delays. Part I: discrete time case," in Proceedings of the 5th International Conference on Control and Automation, Budapest, Hungary, 2005.

[11] H. Zhang, L. Xie, and G. Duan, " $H_{\infty}$ control of discretetime systems with multiple input delays," IEEE Transactions on Automatic Control, vol. 52, no. 2, pp. 271-283, 2007.

[12] B.-L. Zhang, L. Ma, and Q.-L. Han, "Sliding mode $\mathrm{H}_{\infty}$ control for offshore steel jacket platforms subject to nonlinear selfexcited wave force and external disturbanceurbance," Nonlinear Analysis: Real World Applications, vol. 14, no. 1, pp. 163-178, 2013.

[13] B. Zhang, Q. Han, X. Zhang, and X. Yu, "Integral sliding mode control for offshore steel jacket platforms," Journal of Sound and Vibration, vol. 331, no. 14, pp. 3271-3285, 2012.

[14] S. R. Hall and N. M. Wereley, "Performance of higher harmonic control algorithms for helicopter vibration reduction," Journal of Guidance, Control, and Dynamics, vol. 16, no. 4, pp. 793-797, 1993.

[15] S. Han, G. Tang, Y. Chen, and X. Yang, "Optimal vibration control for vehicle active suspension discrete-time systems with actuator time delay," Asian Journal of Control, vol. 15, no. 6, pp. 1579-1588, 2013.

[16] H. Gao, J. Lam, and C. Wang, "Multi-objective control of vehicle active suspension systems via load-dependent controllers," Journal of Sound and Vibration, vol. 290, no. 3-5, pp. 654-675, 2006.

[17] L. Mirkin, "On the approximation of distributed-delay control laws," Systems \& Control Letters, vol. 51, no. 5, pp. 331-342, 2004.

[18] Y. Huang, W. C. Xue, and C. Z. Zhao, "Active disturbance rejection control: methodology and theoretical analysis," Journal of Systems Science and Mathematical Sciences, vol. 31, no. 9, pp. 1111-1129, 2011.

[19] Y. Shi, J. Huang, and B. Yu, "Robust tracking control of networked control systems: application to a networked DC motor," IEEE Transactions on Industrial Electronics, vol. 60, no. 12, pp. 5864-5874, 2013.

[20] X. Guo and M. Bodson, "Analysis and implementation of an adaptive algorithm for the rejection of multiple sinusoidal disturbances," IEEE Transactions on Control Systems Technology, vol. 17, no. 1, pp. 40-50, 2009.

[21] H. Zhao, Q. Chen, and $\mathrm{S} . \mathrm{Xu}$, " $H_{\infty}$ guaranteed cost control for uncertain Markovian jump systems with mode-dependent distributed delays and input delays," Journal of the Franklin Institute, vol. 346, no. 10, pp. 945-957, 2009.

[22] I. D. Landau, M. Alma, J. J. Martinez, and G. Buche, "Adaptive suppression of multiple time-varying unknown vibrations using an inertial actuator," IEEE Transactions on Control Systems Technology, vol. 19, no. 6, pp. 1327-1338, 2011.

[23] M. Shamsuzzoha and M. Lee, "Enhanced disturbance rejection for open-loop unstable process with time delay," ISA Transactions, vol. 48, no. 2, pp. 237-244, 2009. 
[24] L. Guo and S. Cao, "Anti-disturbance control theory for systems with multiple disturbances: a survey," ISA Transactions, vol. 53, no. 4, pp. 846-849, 2014.

[25] X. Li, T. W. S. Chow, and J. K. L. Ho, "Iterative learning control for a class of nonlinear discrete-time systems with multiple input delays," International Journal of Systems Science, vol. 39, no. 4, pp. 361-369, 2008.

[26] J. Zhang, H. Zhang, Y. Luo, and T. Feng, "Model-free optimal control design for a class of linear discrete-time systems with multiple delays using adaptive dynamic programming," Neurocomputing, vol. 135, pp. 163-170, 2014.

[27] G.-Y. Tang and H.-H. Wang, "Suboptimal control for discrete linear systems with time-delay: a successive approximation approach," Acta Automatica Sinica, vol. 31, no. 3, pp. 419-426, 2005.

[28] H. H. Wang and G. Y. Tang, "Observer-based optimal output tracking for discrete-time Systems with multiple state and input delays," International Journal of Control, Automation and Systems, vol. 7, no. 1, pp. 57-66, 2009.

[29] H. T. Banks, M. Q. Jacobs, and M. R. Latina, "The synthesis of optimal controls for linear, time-optimal problems with retarded controls," Journal of Optimization Theory and Applications, vol. 8, pp. 319-366, 1971.

[30] H. Ma, G.-Y. Tang, and Y.-D. Zhao, "Feedforward and feedback optimal control for offshore structures subjected to irregular wave forces," Ocean Engineering, vol. 33, no. 8-9, pp. 1105-1117, 2006. 


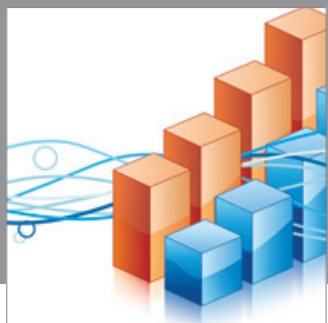

Advances in

Operations Research

mansans

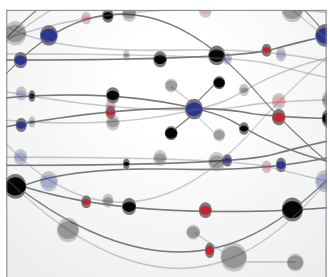

The Scientific World Journal
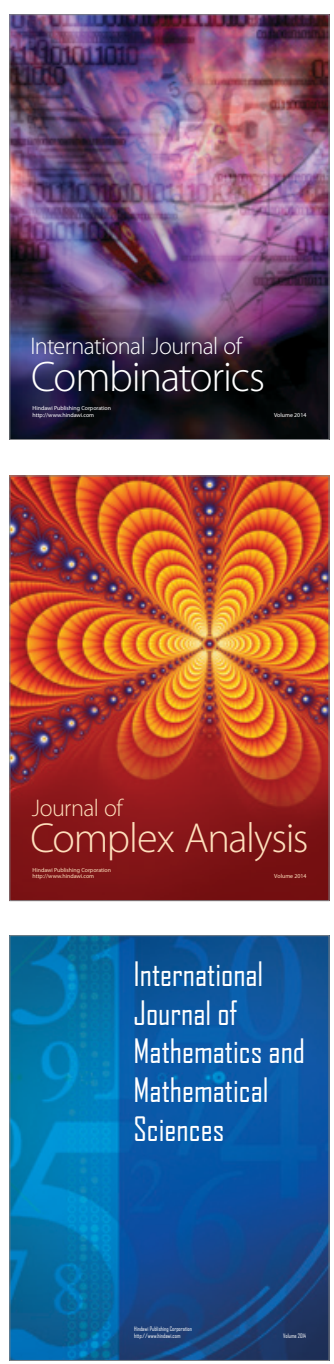
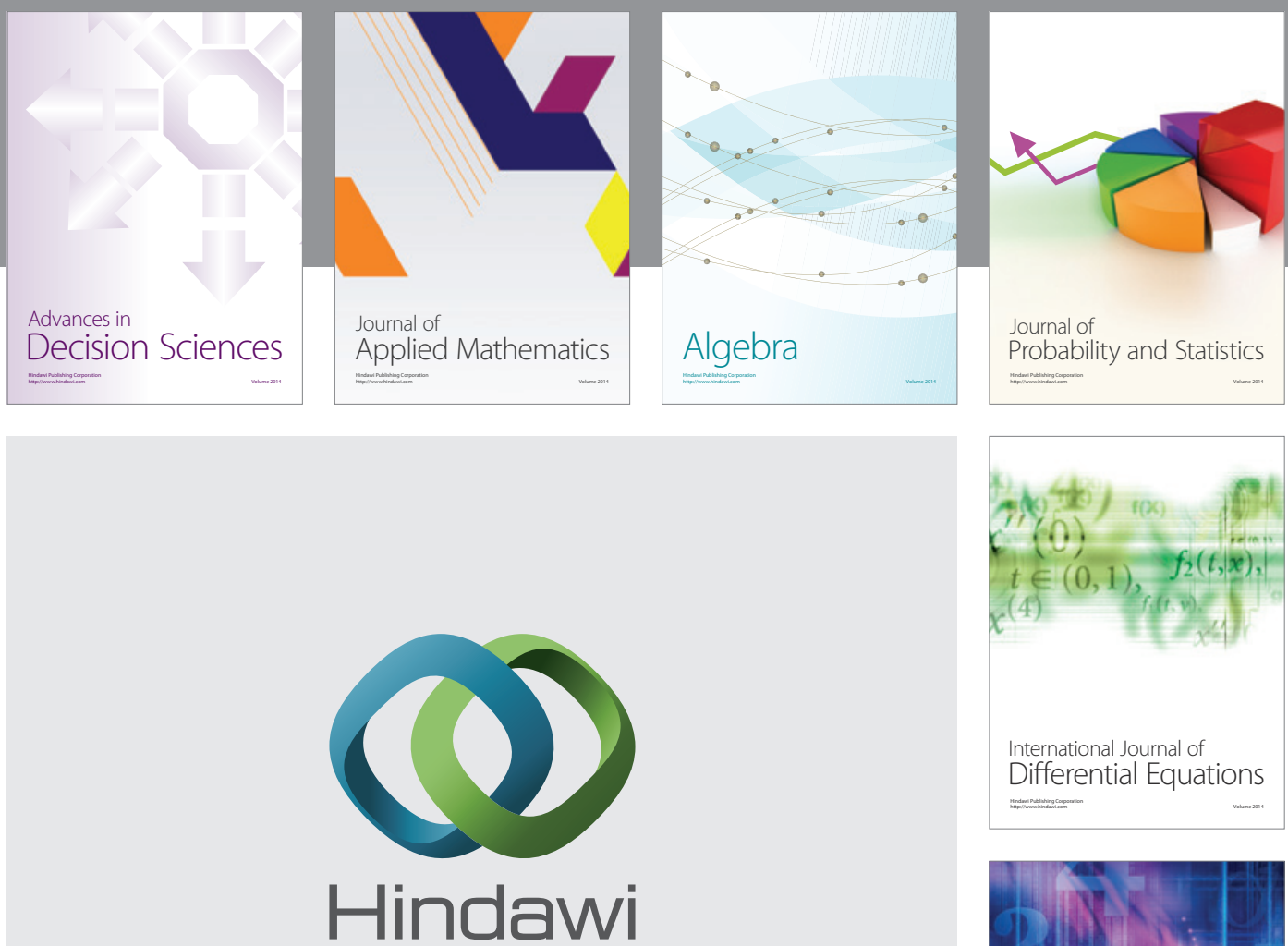

Submit your manuscripts at http://www.hindawi.com
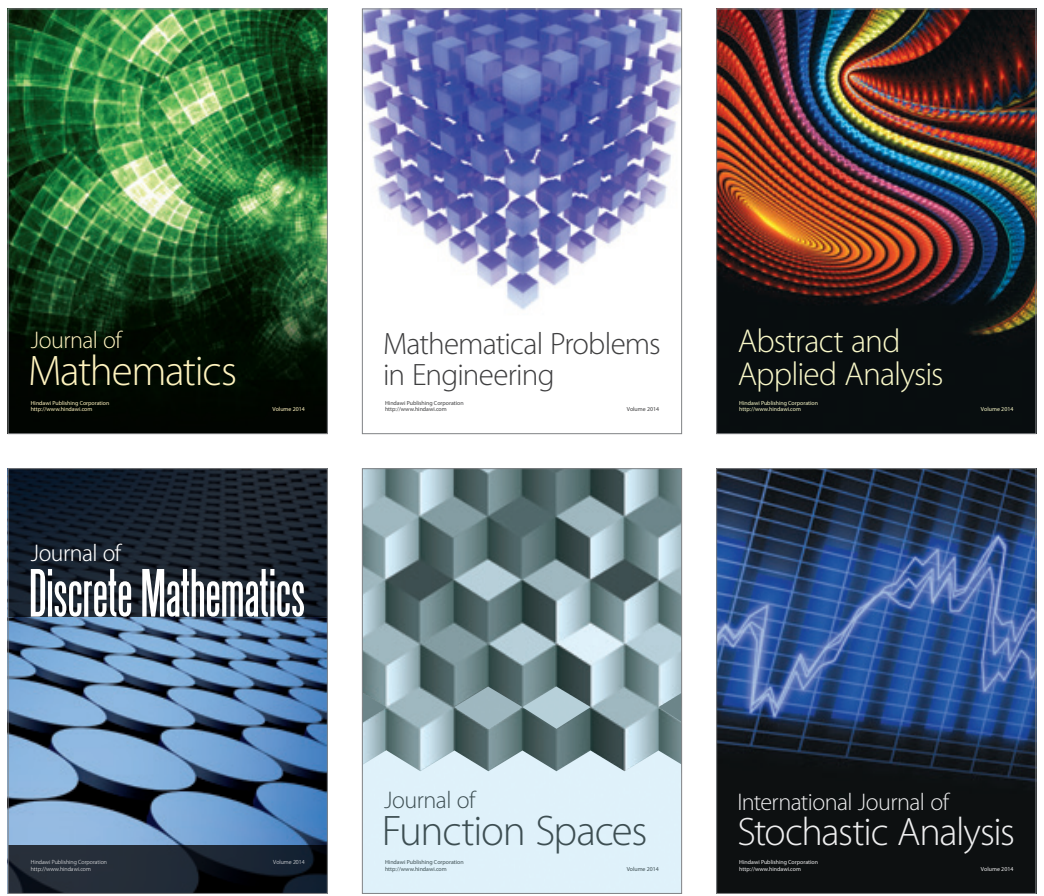

Journal of

Function Spaces

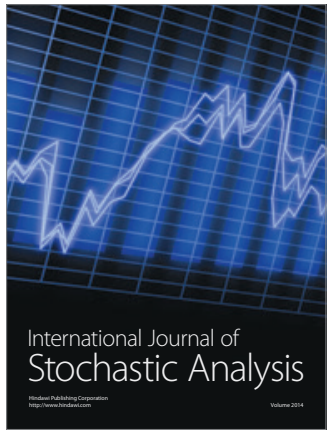

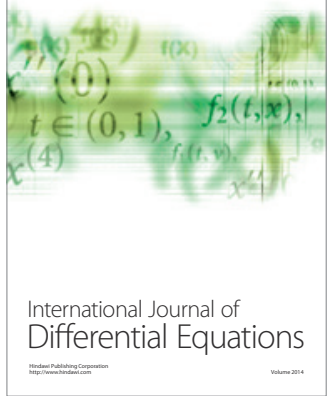
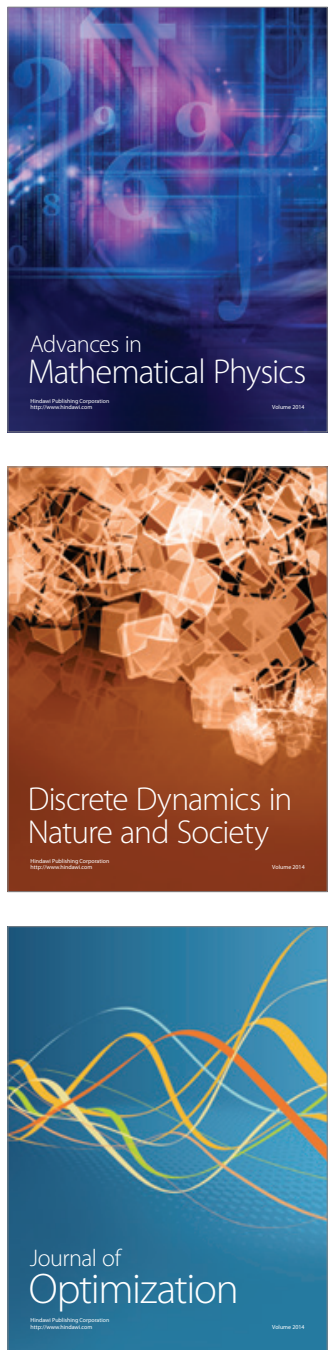